\title{
Universidad Michoacana de San Nicolás de Hidalgo
}

\author{
Juan Serrato Rodríguez,* Alberto Ruiz Marines**
}

RESUMEN: En años recientes el gobierno mexicano a través del CONACYT ha dado un gran impulso al desarrollo de la nanotecnología. La Universidad Michoacana de San Nicolás de Hidalgo a través del Instituto de Investigación en Metalurgia y Materiales ha enfocado esfuerzos de investigación en esta área con un grupo de investigadores de reconocido prestigio. En este documento se hace una breve descripción del Instituto así como de sus contribuciones en nanotecnología, las líneas de investigación que se cultivan y la infraestructura científica disponible para realizar investigación.

PALABRAS CLAVE: Síntesis de nanoestructurados, molienda de alta energía con bolas, sol-gel, nanopartículas de ZnO.

ABSTRACT: In recent years, CONACyT Mexico has dedicated many resources to develop the nanotechnology. The Universidad Michoacana de San Nicolás de Hidalgo through the Instituto de Investigación en Metalurgia y Materiales has focused research efforts in this area with a group of scientist with excellent academic credentials. In this document, a brief description of the Institute as well as its contributions to nanotechnology, research interest and available scientific infrastructure is conducted.

KEYWORDS: Synthesis of nanostructured, high-energy ball milling, sol-gel, ZnO nanoparticles.

\section{Introducción}

El actual Instituto de Investigación en Metalurgia y Materiales (IIMM), de la Universidad Michoacana de San Nicolás de Hidalgo, fue creado en junio de 1972 con el nombre de Instituto de Investigaciones Metalúrgicas (IIM) como una respuesta a la necesidad de realizar investigación y formar recursos de primer nivel en concordancia con el naciente polo de desarrollo en Lázaro Cárdenas "Las Truchas" en la costa michoacana del Océano Pacífico.

En aquella época, el Instituto se estructuró por departamentos y líneas de investigación girando en torno a la metalurgia física, mecánica, química y también en forma general, en torno al aprovechamiento de los recursos naturales. De hecho, el primer programa educativo de posgrado en la Universidad Michoacana se inició en el Instituto en la misma década. Además de

Recibido: 24 de junio de 2016. Aceptado: 23 de agosto de 2016.

* Profesor Investigador Titular C del IIMM. Correspondencia: (jserrato@umich.mx).

** Director del IIMM. Correspondencia: (alruiz@umich.mx). Edificio U, Ciudad Universitaria, Avenida Francisco J. Múgica S/N, Ciudad Universitaria, C.P. 58030 Morelia, Michoacán. 
colaborar vía proyectos de investigación con empresas de la costa michoacana, la actividad más importante de esta dependencia universitaria, se ha centrado en la formación de un alto número de recursos humanos a nivel licenciatura y sobre todo en posgrado, capacitados en metalurgia y materiales.

Actualmente, los programas de estudio de maestría y doctorado que ofrece el IIMM se encuentran reconocidos con Nivel de Consolidados dentro del Padrón Nacional de Posgrados de Calidad (PNPC) del Consejo Nacional de Ciencia y Tecnología (CONACyT). Esto permite que el IIMM pueda otorgar becas CONACyT para estudios de posgrado a estudiantes que cumplan con los requisitos de admisión.

En los últimos años, el estudio de estructuras y materiales en la escala nanométrica ha sido adoptado por varios investigadores de este Instituto, con la tarea de generar fundamentalmente conocimiento científico y aplicaciones útiles e interesantes, principalmente en las líneas de investigación de los materiales cerámicos y metálicos. Las actividades relacionadas con nanociencia datan de hace más de una década, iniciándose de facto con la adquisición de infraestructura de microscopía electrónica de barrido (MEB), de transmisión (MET), de fuerza atómica (FA) y las espectroscopías de análisis dispersivo de rayos $\mathrm{X}$, de pérdida de energía de electrones, de infrarrojo y más recientemente Raman y nanoindentación. Dichas actividades se han intensificado en la última década sobre todo dentro del cuerpo académico consolidado de "Síntesis y Procesamiento de Cerámicas Avanzadas" del mismo Instituto.

De manera simultánea se han establecido relaciones de colaboración con otros cuerpos académicos y con diversas instituciones nacionales y extranjeras.

\section{Infraestructura relevante en nanociencia}

El Instituto cuenta con equipo con tecnología de punta para la investigación en nanociencia en los laboratorios de microscopía electrónica, en particular, el microscopio de barrido de emisión de campo Jeol modelo JSM 7600F y el de transmisión de electrones Tecnai supertwin con emisión de campo, así como el microscopio de fuerza atómica (figura 2), que constituyen una herramienta fundamental en la caracterización de noanoestructuras. El microscopio TEM es un TECNAI super twin de 200KV dotado con modo STEM, además de ser totalmente analítico y con herramientas de espectroscopías EDX y EELS con filtrado de energía EFTEM.

Otras facilidades para estudios en nanotecnología incluyen los laboratorios de espectroscopías de infrarrojo, Raman y nanoindentación; además de los laboratorios de química analítica y otros laboratorios menores acondicionados por los propios investigadores en función de sus actividades cotidianas. 
FIGURA 1. Laboratorios de microscopía electrónica: (izquierda) microscopio electrónico de barrido, y (derecha) microscopio de transmisión de electrones con emisión de campo y microscopio de fuerza atómica.
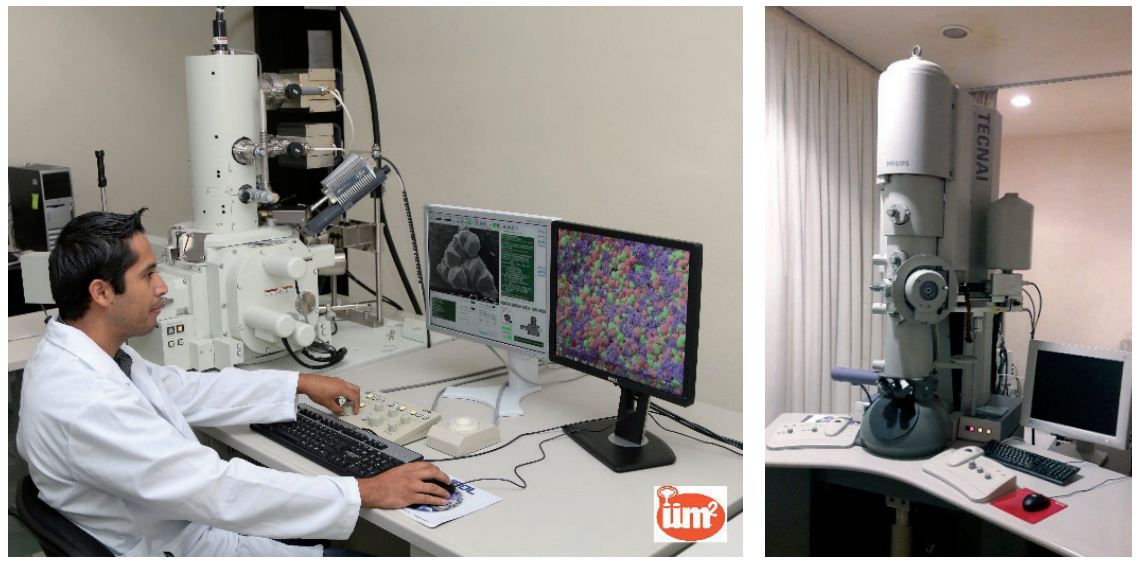

\section{Líneas de investigación relacionadas con nanociencia}

Existen seis líneas de investigación desarrolladas por investigadores de este Instituto. A continuación, se describen en conexión con una muestra de publicaciones recientes sobre nanociencia.

\section{1) Síntesis, caracterización y procesamiento de cerámicas y películas delgadas con aplicaciones industriales y biomédicas}

María Eugenia Contreras et al. (eucontre@umich.mx).

FIGURA 2. Laboratorios de Materiales Cerámicos: (izquierda) nanoindentador TI 700 UBI, y (derecha) microscopio espectrómetro de Raman de alto rendimiento.
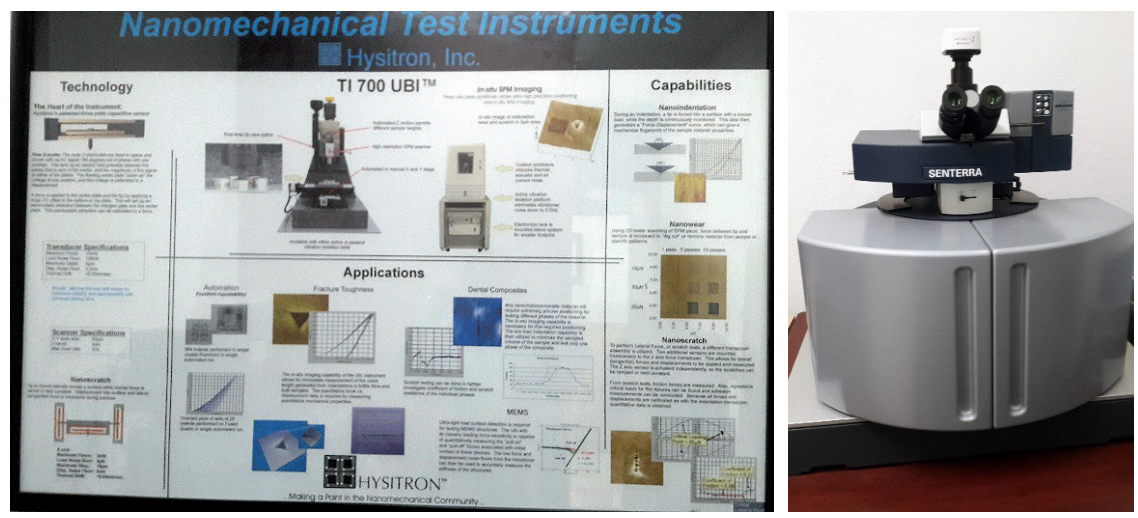
Artículo: Synthesis of nanostructured zirconia electrodeposited films on AISI 316L stainless steel and its behavior in corrosion resistance.

Materials Letters,Vol. 58, 191-195, 2004.

Patente: Proceso para electrodepositar películas de óxido de zirconio sobre acero inoxidable por medio del paso de corriente eléctrica a través de una solución de oxicloruro de zirconio.

No. de Solicitud: PR/E/2003/016937, PA/A/2003/004007, Fecha 07/05/2003. No. de Registro: Título \# 264808.

México, D. F. Aprobada 02/03/2009.

Los trabajos anteriores versan sobre la síntesis de nanopartículas de óxido de zirconio por rutas electroquímicas y su empleo nanoestructurado por depositación catódica para aliviar el problema de la corrosión por picaduras en aceros inoxidables AISI 316L; demostrándose que las nanoestructuras de zirconia electro depositadas en forma de películas uniformes disminuyen la densidad de corriente por debajo de los valores obtenidos en aceros inoxidables AISI 316L sin recubrir, y por tanto disminuyen considerablemente la corrosión en los aceros, según se refiere en la patente respectiva.

\section{2) Sintesis de nanopartículas}

Gerardo Rosas et al. (grtrejo@umich.mx).

a) Synthesis of gold nanoparticles with different atomistic structural characteristics.

Materials Characterization, 58 (8), 694-700, 2007.

b) Iron nanoparticles produced by high-energy ball milling.

Journal of Nanoparticle Research, 9 (5), 945-950, 2007.

Los autores utilizan un proceso de reducción química de una sal de oro para producir nanopartículas de diversos tamaños y por tanto configuraciones atómicas diversas. La caracterización incluye técnicas digitales experimentales de microscopía electrónica de alta resolución comparadas con imágenes simuladas con base en la teoría dinámica de difracción electrónica y a cálculos mecánicos moleculares cuánticos de la teoría funcional de densidad. Concluyéndose que la estructura electrónica de las nanopartículas es significativamente afectada por sus configuraciones atómicas, induciéndose una mayor actividad catalítica para configuraciones decahedrales en las que las caras de simetría cinco son expuestas.

En una ruta física, los mismos autores sintetizan nanopartículas de hierro usando molinos de alta energía, haciendo la caracterización estructural mediantes microscopía electrónica de alta resolución llegando a la conclusión 
de que la estructura de la fase mayoritaria corresponde a nanopartículas de hierro con estructura fcc.

\section{3) Caracterización de nanomateriales}

Ariosto Medina et al. (ariosto@umich.mx).

a) High-resolution transmission electron microscopy and molecular simulation analysis of $\mathrm{Fe}_{2-3} \mathrm{~N}$ and $\mathrm{Fe}_{4} \mathrm{~N}$ formation for a nitrided 4140 steel.

Revista de Metalurgia, 04/2015.

b) Characterization of $\mathrm{ZnO}$ nanoparticles with short-bar shape produced by chemical precipitation.

Materials Letters. Vol. 71. pp. 81-83, 2012.

En la tercera línea de investigación, y trabajando con procesos de nitruración para endurecimiento de aceros, los autores caracterizan las interfases endurecidas por técnicas de microscopía electrónica de alta resolución, encontrándose la formación de diversas nanofases cristalinas, entre otras, la fase $\gamma$-Fe4N compuesta por nanopartículas cúbicas que crecen en la dirección $<001>$ y presumiblemente juegan un papel preponderante en las propiedades mecánicas de los aceros.

En otro trabajo, los mismos autores utilizan un procedimiento de síntesis de nanopartículas de óxido de zinc por precipitación química, y el producto de síntesis lo caracterizan mediante técnicas de difracción de rayos $\mathrm{X}$, microscopía electrónica de barrido y de transmisión de emisión de campo y de alta resolución, tipificando una estructura tipo wurtzita que cristaliza en la dirección [0001].

\section{4) Síntesis de nanopartículas por precipitación química homogénea y procesamiento cerámico para recubrimientos resistentes al choque térmico y antiabrasivos}

Juan Zarate et al. (jzarate@umich.mx).

a) Synthesis and mechanical characterization by nanoindentation of polycrystalline YAG with Eu and Nd additions.

Ceramics International, 39, 3141-3149, 2013.

b) Microstructure and mechanical properties of $\mathrm{Al}_{2} \mathrm{O}_{3}-\mathrm{YSZ}$ spherical polycrystalline composites.

Journal of the European Ceramic Society, 33, 1907-1916, 2013.

Nanopolvos cerámicos YAG dopados y sin dopar fueron sintetizadas químicamente a través de un ruta tipo Pechini pero modificada, dichos nanopolvos fueron sujetos a secado por aspersión y densificados a temperaturas tan 
bajas como $1550^{\circ} \mathrm{C}$ en aire. El parámetro de red se incrementó debido a los dopantes de europio y neodimio adicionados. La nanodureza fue de $22 \mathrm{GPa}$, el módulo elástico fue de $315 \mathrm{GPa}$; estos elevados valores en las propiedades mecánicas son comparables a los reportados para cristales únicos.

En la otra investigación, los autores usaron polvos nanométricos de alúmina y zirconia estabilizada con ytria para producir compósitos con alta resistencia a la tenacidad.

\section{5) Estudio de fenómenos coloidales e interfaciales en metalurgia extractiva}

Feng RAO et al. (fengrao@umich.mx).

a) Synthesis and characterization of Ag-PILC through the formation of $\mathrm{Ag}$ montmorillonite nano-composite.

Nano, 10, 31-39, 2015.

b) Synthesis of Ag-pilc through direct insertion of silver nanoparticles into smectite interlayers.

Materials Technology, 27, 186-190, 2012.

En esta línea de investigación, se desarrolló un nuevo método de síntesis de arcillas pilareadas con plata mediante el cual las nanopartículas de plata se formaron en las intercapas de arcillas montmoriloníticas. Se partió de iones de plata que fueron intercambiados en las intercapas de montmorilonita y una vez ahí, fueron reducidos a nanopartículas de plata usando citrato trisódico a $100^{\circ} \mathrm{C}$. El nanocompósito resultante fue caracterizado por XRD, TEM y SSA(BET). Las nanopartículas confirieron mayor estabilidad térmica y estructural. Considerando que las variables de síntesis pueden manipularse, por tanto, es posible modificar la porosidad y los nanospilares.

En otro enfoque para preparar arcillas pilareadas con plata, las nanopartículas se insertan directamente entre las capas de esmectita, como una alternativa de mayor estabilidad estructural comparada con el método convencional de intercambio policatiónico. En esta propuesta, las cargas negativas esmectíticas se cambian a positivas a través de adsorción de especies hidrolizadas de aluminio, mismas que atraen cargas negativas de nano partículas de plata resultando en la formación de un compuesto intercalado, el cual después de calcinarse y caracterizarse por área superficial específica, resulta su uso potencial como catalizador de reacciones orgánicas, o como adsorbente de alta actividad microbiana en tratamiento de aguas. 


\section{6) Mecanismos de nanoestructuramiento utilizando síntesis sol-gel/ tratamiento hidrotermal en óxidos cerámicos semiconductores con impacto en energías renovables}

Juan Serrato et al. (jserrato@umich.mx).

a) Development of nanostructure in ultra-fine anatase powders derived by the low temperature sol-gel solvothermal process.

Journal of Ceramic Processing Research, Vol. 16: 499-504, No. 5, 2015.

b) Nanostructuring anatase through the addition of acetic acid by the solgel low temperature aqueous processing.

Ceramics International, Vol. 40: 8631-8635, No. 6, julio, 2014.

Esta última línea de investigación tiene que ver con el control estructural a nivel nano en materiales de titania dopada y sin dopar sintetizados por la ruta sol-gel de baja temperatura con o sin tratamiento hidrotermal de forma tal que permita controlar los parámetros estructurales de la titania. Los materiales ultra finos obtenidos corresponden a diámetros inferiores a los $6 \mathrm{~nm}$ y el foco de la investigación comprende los mecanismos de engrosamiento del tamaño de partícula; evidenciándose la orientación cristalográfica como principal mecanismo de crecimiento de nanocristales.

En otro enfoque químico del mismo trabajo en que se usó espectroscopía infrarroja para el control del ligando bidentado de titanio, se formaron agentes quelantes vía adiciones sucesivas de ácido acético durante un proceso sol-gel ácido acuoso de baja temperatura, para producir nanopolvos de titania fase anatasa y de alta área específica. Presumiblemente los ligandos formados controlan el desarrollo nanoestructural por varias vías, limitan el crecimiento cristalino particularmente en conexión con las facetas cristalográficas de alta energía. Por otra parte, al aumentar la concentración del ligando se induce una mayor cristalinidad en la anatasa y, finalmente, también se retarda la temperatura de transición anatasa-rutilo.

\section{Logros}

Los logros en la investigación de nanomateriales referida anteriormente, se pueden resumir en el contexto del diseño de métodos prácticos derivados como conclusión de estudios experimentales para aprovechar las bondades de la dimensión nano; como en el caso de la utilización de nanopartículas de zirconia para aumentar la resistencia a la corrosión de aceros AISI 316 L. También, como en el caso del uso de nanopartículas de plata para producir arcillas pilareadas de mayor estabilidad térmica y estructural o bien el uso puente de las mismas nanopartículas para formar compuestos intercalados de alta área específica. La síntesis de nanopartículas por diversos métodos 
químicos y físicos ha permitido acceder al empleo de las mismas en otros experimentos actualmente en progreso.

En otro enfoque químico original sobre el estudio del control de la formación de nanoestructuras en materiales de titania, se logró controlar cuantitativamente el tamaño de partícula, la cristalinidad y la transición de fase anatasa-rutilo vía formación de complejos bidentados. Lo cual constituye un mejor entendimiento del control estructural en investigación nano, según lo reporta la agencia noticiosa Better Understanding Through Technology \& Emerging Research (Elsevier Sci Ltd, The Boulevard, Longford Lane, Kidlington, Oxford OX5 1GB, Oxon, England. Journal of Technology, 1943, ISSN: 1944-1886, BUTTER ${ }^{\mathrm{TM}}$ ID: 0080715).

\section{Docencia y formación de recursos humanos}

En el IIMM la mayoría de los investigadores se desempeñan en el área de NyN, están especialmente comprometidos con actividades de investigación, docencia y formación de recursos humanos a través de la impartición de cursos especializados frente a grupo y dirigiendo tesis de licenciatura, maestría y doctorado, con una gran participación en comités tutorales y como sinodales en exámenes de grado. Participan también en actividades como verano científico, siendo responsables de prácticas profesionales y servicios sociales, brindado asesoría especializada. En actividades administrativas colaborando en la creación y actualización de planes de estudio y en la elaboración y aplicación de exámenes de admisión y cursos propedéuticos entre otras actividades.

La mayor parte de los cursos a nivel licenciatura se imparten en el IIMM y en la nueva carrera de licenciatura en innovación tecnológica de materiales en la cual se imparten materias relacionadas con el área de los nanomateriales.

La formación de recursos humanos en el área de $\mathrm{NyN}$ y áreas afines se ve reflejada en la conclusión de aproximadamente 10 tesis de licenciatura, 30 de maestría y cinco de doctorado, constituyendo el logro más importante por el impacto en la sociedad con la generación de recursos humanos de gran calidad. 